\title{
Transient behavior of water transport in the membrane of a PEM fuel cell
}

\author{
Falin Chen ${ }^{\mathrm{a}, *}$, Yu-Guang Su ${ }^{\mathrm{a}}$, Chyi-Yeou Soong ${ }^{\mathrm{b}}$, Wei-Mon Yan ${ }^{\mathrm{c}}$, Hsin-Sen Chu ${ }^{\mathrm{d}}$ \\ a Institute of Applied Mechanics, National Taiwan University, Taipei 106, Taiwan, ROC \\ ${ }^{\mathrm{b}}$ Department of Aeronautical Engineering, Feng Chia University, Seatwen, Taichung 40745, Taiwan, ROC \\ c Department of Mechatronic Engineering, Huafan University, Shih-Ting, Taipei 22305, Taiwan, ROC \\ d Department of Mechanical Engineering, National Chiao Tung University, Hsin-Chu 300, Taiwan, ROC
}

Received 10 August 2003; received in revised form 14 October 2003; accepted 7 November 2003

\begin{abstract}
The dynamic performance of a PEM fuel cell is one of the most important criteria in the design of fuel cells, especially when the application of the fuel cell in mobile systems is concerned. To attack this issue, we extend the theoretical model developed by Okada (J. Electroanal. Chem. 465 (1999) 1,18) to an unsteady state model and investigate the transient behavior of water transport across the membrane as well as the influences of several physical parameters on the characteristic time to reach the steady state. We also consider the influence due to the presence of foreign impurity ions, which turn out to be a crucial factor affecting the unsteady state features of water transport across the membrane. The results suggest that a higher initial current density to start the operation, a higher water flux from the anode side, a smaller operational current density, and a lower level of contamination in the membrane (especially at the cathode side) can all result in a shorter time for the water transport to reach the steady state, and thus a better dynamic performance of the fuel cell can be obtained.
\end{abstract}

(c) 2003 Elsevier B.V. All rights reserved.

Keywords: PEM fuel cell; Membrane; Water management; Dynamic performance; Unsteady state

\section{Introduction}

In applications of PEM fuel cells in mobile systems, various characteristic times influence the dynamic performance of the power system. Of these, the time interval from the start of operation to the achievement of the steady state of water transport across the membrane is one of the important characteristic times. To seek this time interval and other potential time constants, the unsteady state water management of the fuel cell will be considered. This, in fact, in the last two decades has become one of the crucial issues in PEM fuel cell research, mainly because the electric conductivity depends significantly on the water content in the fuel cell, especially in the membrane [1-6].

\footnotetext{
${ }^{*}$ Corresponding author. Tel.: +886-2-3366-5692; fax: +886-2-33665691.

E-mail address: falin@spring.iam.ntu.edu.tw (F. Chen).
}

Many investigations have developed theoretical models governing water transportation in the components of the fuel cell, such as the fuel gas channel, the gas diffusion layers, the catalyst layers, and the membrane. Together with the boundary conditions on both boundaries and interfaces, nevertheless, the whole mathematical formulation becomes too complicated and tedious to be solved. To avoid the complication and heavy computational loading, a one-dimensional model simulating water transport in the direction across the membrane was developed, see for example [7-11]. The authors of these papers concluded that, under a proper water-balance operation, the important operation parameters could be reasonably estimated. They indicated further that the ohmic loss becomes significant in the high current density regime, while in a moderate current density regime, humidification at the cathode may not be necessary because the water generated at the cathode may be sufficient to counteract the water depletion in the membrane. By considering the non-isothermal effect, 
they pointed out that a high operational temperature might lead to a more serious dehydration of the membrane, increasing its internal resistance.

Later, many other investigations [12-16] extended the model to two dimensions. It was found that the twodimensional simulation results in a lower output voltage than the one-dimensional model. It was indicated further that for a high current density, the ohmic loss in the membrane accounts for a majority part of the overall voltage loss of the cell, and the water diffusing from cathode to anode is not sufficient to supply the need for water at the anode. It was also found that the pressuregradient driven water transportation across the membrane has a significant influence on the water content of the cell and there is an obvious thermal gradient across the cell, which has a significant effect on the cell performance.

Instead of considering all the physical variations in the fuel cell, many studies focused on water management in the whole physical domain including the gas diffusion layer, catalyst layer, and the membrane [17-23], while some work was restricted to the membrane [24,25]. Recently, Okada [26,27] proposed a one-dimensional water transport model in the membrane, in which special attention was paid to the influences imposed by the contamination at both the anode and cathode. Okada pointed out that the water transportation is influenced significantly by the presence of foreign impurity ions, and that, at the cathode, these influences have a greater effect than at the anode. The contaminant may either exist in the membrane before use or enter into the membrane with the fuel due to the corrosion of piping or stack materials. In their series of studies, Okada and coworkers [28-35] found that for a Nafion $\mathscr{R}^{\mathscr{R}}$ membrane, the attraction of some impurity ions is larger than that of the $\mathrm{H}^{+}$cation, and the sequence is $\mathrm{Fe}^{3+}>\mathrm{Ca}^{2+}>\mathrm{Ni}^{2+}>\mathrm{Cu}^{2+}>\mathrm{Na}^{+}$, which implies that an ion of higher valence has a greater attraction to the membrane. Since the conductivity of the membrane is linearly proportional to the number of $\mathrm{H}^{+}$ions in the membrane, the conductivity of the membrane decreases as the number of impurity ions increases. Okada and coworkers indicated further that, since the water content, the number of $\mathrm{H}^{+}$cations and the water permeability all decrease with increasing number of impurity ions, the contamination could actually influence the fuel cell performance seriously. The electro-osmotic effect of the membrane becomes more significant when foreign impurity ions are present and are increasing. Accordingly water transport from the anode to cathode increases. However, on the other hand, the water diffusion decreases due to the presence of impurity ions and water transport from the cathode to anode decreases. Consequently, when the membrane is contaminated, water depletion at the anode will become more serious, leading to a decrease of the cell performance.
In their series of studies [24-35], Okada and coworkers investigated only the steady state water distributions in the membrane. In the application of a fuel cell for a dynamic system, such as a vehicular power system, the transient behavior of the fuel cell operation may be crucial, and this behavior has a close bearing on some of the characteristic times of the system, such as the time needed for water transport in the membrane to reach a steady state. Accordingly, in the present paper, we extend the theoretical model developed by Okada $[26,27]$ and investigate the unsteady state features of the water transportation in the membrane. Special attention is paid to the influence of the relevant physical and operational parameters on the characteristic time for the water transport across the membrane to reach the steady state.

The paper is organized as follows. In Section 2 the mathematical model and relevant boundary conditions regarding the unsteady state water transport across the membrane are presented. In Section 3 the values of the relevant physical parameters are given. In Section 4 the results from analyses regarding the transient behavior of water transport across the membrane and the time needed for the water distribution to reach the steady state are discussed. Finally, in Section 5 the conclusions are presented.

\section{Theoretical model of unsteady water transport in membrane}

Consider a membrane of PEM fuel cell of thickness $d$ (Fig. 1). We investigate the unsteady state water transport across the membrane. Although the theoretical model is essentially an extension from the model of Okada [26,27], we still present the details of the mathematical formulation and relevant physical parameters in this section for the convenience of the following discussion. Firstly, we assume that the interfaces between the membrane and the two electrodes are flat planes and all the physical properties are uniform on the plane. The volume of the membrane is assumed to be unchanging

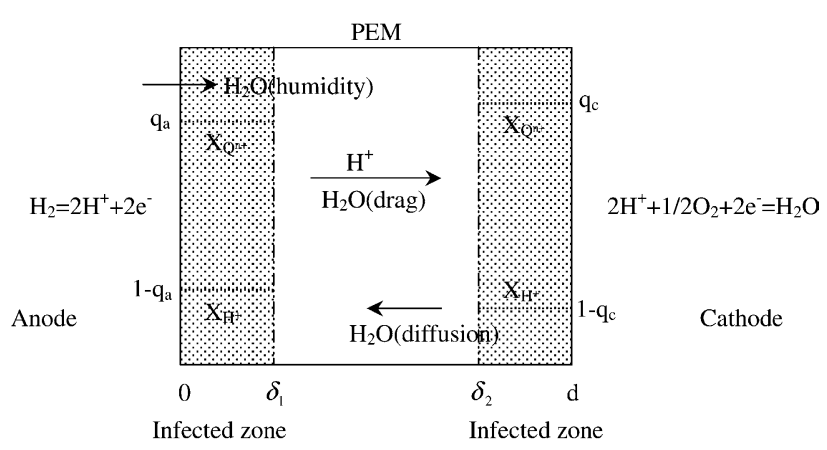

Fig. 1. A schematic description of the physical domain considered. 
with the variation of hydration, so that the thickness $d$ remains constant. The reactions in electrodes are assumed to be much faster than the water transport in the membrane, so that the current density across the electrodes and the membrane is a step function. Since the combination of the diffusion and the electro-osmosis effects accounts for the flux of water [24], namely,

$J=-D \frac{\partial c}{\partial x}+\frac{j}{F} w$

so that the water transport equation in the membrane can be written as

$\frac{\partial c}{\partial t}=-\frac{\partial J}{\partial x}=\frac{\partial}{\partial x}\left(D \frac{\partial c}{\partial x}-\frac{j}{F} w\right)$,

in which $J$ is the flux of water across the membrane, $D$ is the diffusion coefficient of water in the membrane, $c$ is the water concentration in the membrane, $i$ is the current density across the membrane, $w$ is the water transference coefficient, and $F$ is the Faraday constant. To account for the fact that $D$ and $w$ change with the water concentration, these two coefficients are expressed by the following functional relations [26,27]

$D=D^{(0)}+D^{(1)} c+D^{(2)} c^{2}+\cdots$,

$w=w^{(0)}+w^{(1)} c+w^{(2)} c^{2}+\cdots$.

After substituting (3) and (4) into (2), we obtain

$$
\begin{aligned}
\frac{\partial c}{\partial t}= & {\left[D^{(0)} \frac{\partial^{2} c}{\partial x^{2}}-\frac{j}{F} w^{(1)} \frac{\partial c}{\partial x}\right] } \\
& +\left[D^{(1)} c \frac{\partial^{2} c}{\partial x^{2}}+D^{(1)}\left(\frac{\partial c}{\partial x}\right)^{2}-\frac{2 j}{F} w^{(2)} c \frac{\partial c}{\partial x}\right]+\cdots
\end{aligned}
$$

We neglect the higher order terms on the right hand side of (5) and obtain the final water transport equation as follows

$\frac{\partial c}{\partial t}=D^{(0)} \frac{\partial^{2} c}{\partial x^{2}}-\frac{j}{F} w^{(1)} \frac{\partial c}{\partial x}$.

As shown in Fig. 1, the membrane is divided into three regions. The left region extends from $x=0$ to $\delta_{1}$, in which the contaminant ion designated as $\mathrm{Q}^{n+}$ comes from the anode, and is called the left infected zone (or the Q-type membrane). The middle region extends from $x=\delta_{1}$ to $\delta_{2}$, in which no contaminant ion appears, and is called the non-infected zone (or the H-type membrane). The right region extends from $x=\delta_{2}$ to $d$, in which the contaminant ion comes from the cathode, and is called the right infected zone. In the present study, the distributions of $\mathrm{Q}^{n+}$ in the two infected zones are assumed to be uniform, so that the ionic fractions of $\mathrm{Q}^{n+}$ and $\mathrm{H}^{+}$, denoted by $X_{\mathrm{Q}^{n+}}$ and $X_{\mathrm{H}^{+}}$, respectively, of these three zones can be expressed by

$X_{\mathrm{Q}^{n+}}=q_{\mathrm{a}}, \quad X_{\mathrm{H}^{+}}=1-q_{\mathrm{a}} \quad\left(0<x<\delta_{1}\right)$,
$X_{\mathrm{Q}^{n+}}=0, \quad X_{\mathrm{H}^{+}}=1 \quad\left(\delta_{1}<x<\delta_{2}\right)$,

$X_{\mathrm{Q}^{n+}}=q_{\mathrm{c}}, \quad X_{\mathrm{H}^{+}}=1-q_{\mathrm{c}} \quad\left(\delta_{2}<x<d\right)$,

in which $q_{\mathrm{a}}$ is the ionic fraction of $\mathrm{Q}^{n+}$ at the anode $\left(0<q_{\mathrm{a}}<1\right)$ and $q_{\mathrm{c}}$ is the ionic fraction of $\mathrm{Q}^{n+}$ at the cathode $\left(0<q_{\mathrm{c}}<1\right)$, and $X_{\mathrm{Q}^{n+}} X_{\mathrm{H}^{+}}$are expressed by $[26,27,35]$

$X_{\mathrm{Q}^{n+}}=\frac{n c_{\mathrm{Q}^{n+}}}{n c_{\mathrm{Q}^{n+}}+c_{\mathrm{H}^{+}}}, \quad X_{\mathrm{H}^{+}}=\frac{c_{\mathrm{H}^{+}}}{n c_{\mathrm{Q}^{n+}}+c_{\mathrm{H}^{+}}}$,

in which $c_{\mathrm{Q}^{n+}}$ and $c_{\mathrm{H}^{+}}$are respectively the concentration of $\mathrm{Q}^{n+}$ and $\mathrm{H}^{+}$and $n$ is the valence of the contaminant ion.

In the two infected zones, the diffusion coefficient of water $D$ is modified by the presence of the contaminants as follows [26,27,31,32]

$D=X_{\mathrm{H}^{+}} D_{\mathrm{HM}}+X_{\mathrm{Q}^{n+}} D_{\mathrm{QM}}$,

in which $D_{\mathrm{HM}}$ is the diffusion coefficient of water of the H-type membrane and $D_{\mathrm{QM}}$ is the water diffusion coefficient of the Q-type membrane. Similarly, with contamination, the water transference coefficient is modified into [26,27,31-35]

$w=N_{\mathrm{H}^{+}} w_{\mathrm{HM}}+N_{\mathrm{Q}^{n+}} w_{\mathrm{QM}}$,

where $N_{\mathrm{H}^{+}}$and $N_{\mathrm{Q}^{n+}}$ are respectively the ionic transference numbers of $\mathrm{H}^{+}$and $\mathrm{Q}^{n+}$ ions in the membrane, defined as

$N_{\mathrm{H}^{+}}=\frac{u_{\mathrm{H}^{+}} X_{\mathrm{H}^{+}}}{u_{\mathrm{H}^{+}} X_{\mathrm{H}^{+}}+u_{\mathrm{Q}^{n+}} X_{\mathrm{Q}^{n+}}}=\frac{X_{\mathrm{H}^{+}}}{X_{\mathrm{H}^{+}}+p X_{\mathrm{Q}^{n+}}}$,

$N_{\mathrm{Q}^{n+}}=1-N_{\mathrm{H}^{+}}=\frac{p X_{\mathrm{Q}^{n+}}}{X_{\mathrm{H}^{+}}+p X_{\mathrm{Q}^{n+}}}$.

In the above equations, $w_{\mathrm{HM}}$ and $w_{\mathrm{QM}}$ are the water transference coefficients of the H-type and Qtype membrane, respectively, $u_{\mathrm{H}^{+}}$and $u_{\mathrm{Q}^{n+}}$ are the mobility of $\mathrm{H}^{+}$and $\mathrm{Q}^{\mathrm{n}+}$, respectively, and $p=u_{\mathrm{Q}^{n+}} /$ $u_{\mathrm{H}^{+}}<1$.

Due to the presence of contaminants, the water transport equations of the three regions are also changed into the following

$$
\begin{array}{ll}
\frac{\partial c}{\partial t}=D_{q_{\mathrm{a}}}^{(0)} \frac{\partial^{2} c}{\partial x^{2}}-\frac{j}{F} w_{q_{\mathrm{a}}}^{(1)} \frac{\partial c}{\partial x} & \left(0<x<\delta_{1}\right), \\
\frac{\partial c}{\partial t}=D_{\mathrm{HM}}^{(0)} \frac{\partial^{2} c}{\partial x^{2}}-\frac{j}{F} w_{\mathrm{HM}}^{(1)} \frac{\partial c}{\partial x} & \left(\delta_{1}<x<\delta_{2}\right), \\
\frac{\partial c}{\partial t}=D_{q_{\mathrm{c}}}^{(0)} \frac{\partial^{2} c}{\partial x^{2}}-\frac{j}{F} w_{q_{\mathrm{c}}}^{(1)} \frac{\partial c}{\partial x} & \left(\delta_{2}<x<d\right),
\end{array}
$$

in which $D_{q_{\mathrm{a}}}^{(0)}, D_{\mathrm{HM}}^{(0)}$ and $D_{q_{\mathrm{c}}}^{(0)}$ are respectively zero-order coefficients with respect to $c$ of the water diffusion coefficients (Eq. (3)) of the left, the middle, and the right zones (see Fig. 1), and $w_{q_{\mathrm{a}}}^{(1)}, w_{\mathrm{HM}}^{(1)}$ and $w_{q_{\mathrm{c}}}^{(1)}$ are the first-order coefficients with respect to $c$ of the water transference coefficients (Eq. (4)) of these three zones, 
respectively. According to Eqs. (3), (7a), (7c) and (9), $D_{q_{\mathrm{a}}}^{(0)}$ and $D_{q_{\mathrm{c}}}^{(0)}$ can be expressed by

$$
\begin{aligned}
D_{q_{\mathrm{a}}}^{(0)} & =X_{\mathrm{H}^{+}} D_{\mathrm{HM}}^{(0)}+X_{\mathrm{Q}^{n+}} D_{\mathrm{QM}}^{(0)} \\
& =\left(1-q_{\mathrm{a}}\right) D_{\mathrm{HM}}^{(0)}+q_{\mathrm{a}} D_{\mathrm{QM}}^{(0)} \quad\left(0<x<\delta_{1}\right),
\end{aligned}
$$

$$
\begin{aligned}
D_{q_{\mathrm{c}}}^{(0)} & =X_{\mathrm{H}^{+}} D_{\mathrm{HM}}^{(0)}+X_{\mathrm{Q}^{n+}} D_{\mathrm{QM}}^{(0)} \\
& =\left(1-q_{\mathrm{c}}\right) D_{\mathrm{HM}}^{(0)}+q_{\mathrm{c}} D_{\mathrm{QM}}^{(0)} \quad\left(\delta_{2}<x<d\right),
\end{aligned}
$$

in which $D_{\mathrm{QM}}^{(0)}$ is the zeroth-order coefficient of the water diffusion coefficient of the Q-type membrane. From Eqs. (4), (7a), (7c), (10) and (11), $w_{q_{\mathrm{a}}}^{(1)}$ and $w_{q_{\mathrm{c}}}^{(1)}$ can be rewritten as

$$
\begin{aligned}
w_{q_{\mathrm{a}}}^{(1)} & =\frac{1}{1-q_{\mathrm{a}}+p_{\mathrm{a}} q_{\mathrm{a}}}\left\{\left(1-q_{\mathrm{a}}\right) w_{\mathrm{HM}}^{(1)}+p_{\mathrm{a}} q_{\mathrm{a}} w_{\mathrm{QM}}^{(1)}\right\} \\
(0 & \left.<x<\delta_{1}\right), \\
w_{q_{\mathrm{c}}}^{(1)} & =\frac{1}{1-q_{\mathrm{c}}+p_{\mathrm{c}} q_{\mathrm{c}}}\left\{\left(1-q_{\mathrm{c}}\right) w_{\mathrm{HM}}^{(1)}+p_{\mathrm{c}} q_{\mathrm{c}} w_{\mathrm{QM}}^{(1)}\right\} \\
\left(\delta_{2}\right. & <x<d),
\end{aligned}
$$

in which $w_{\mathrm{QM}}^{(1)}$ is the first-order coefficient of the water transference coefficient of the Q-type membrane and $p_{\text {a }}$ is the ratio between the mobilities of $Q^{n+}$ and $\mathrm{H}^{+}$in the membrane close to the anode and $p_{\mathrm{c}}$ is another mobility ratio in the membrane close to the cathode.

To examine the unsteady state features of the water transport in the membrane, we assume initially that the water concentration is

$$
c(x, 0)=c_{0}(x) \quad(0<x<d ; t=0),
$$

in which $c_{0}(x)$ is the water concentration corresponding to the initial current density $j_{0}$. In addition, there are four boundaries in the physical domain (Fig. 1), and the relevant boundary conditions are illustrated in the following:

(1) The interface between the anode and the left infected zone $(x=0)$. At this interface, the continuity of water flux is satisfied so that

$$
k\left[c_{\mathrm{a}}-c(0, t)\right]=-D_{q_{\mathrm{a}}}^{(0)} \frac{\partial c(0, t)}{\partial x}+\frac{j}{F}\left[w_{q_{\mathrm{a}}}^{(0)}+w_{q_{\mathrm{a}}}^{(1)} c(0, t)\right],
$$

in which $k$ is the rate constant of Henry's law accounting for the water flux entering into or emerging from the membrane driven by the water concentration gradient in the membrane, called the humidification parameter, $c_{\mathrm{a}}$ is the water concentration in the membrane being in equilibrium with the water vapor in the anode gas.

(2) The interface between the left infected zone and the non-infected zone $\left(x=\delta_{1}\right)$. At this interface the continuities of both water flux and water concentration are satisfied, namely,

$$
\begin{aligned}
- & D_{q_{\mathrm{a}}}^{(0)} \frac{\partial c\left(\delta_{1-}, t\right)}{\partial x}+\frac{j}{F}\left[w_{q_{\mathrm{a}}}^{(0)}+w_{q_{\mathrm{a}}}^{(1)} c\left(\delta_{1-}, t\right)\right] \\
= & -D_{\mathrm{HM}}^{(0)} \frac{\partial c\left(\delta_{1+}, t\right)}{\partial x}+\frac{j}{F}\left[w_{\mathrm{HM}}^{(0)}+w_{\mathrm{HM}}^{(1)} c\left(\delta_{1+}, t\right)\right],
\end{aligned}
$$

$c\left(\delta_{1-}, t\right)=c\left(\delta_{1+}, t\right)$,

where $\delta_{1+}$ and $\delta_{1-}$ account for the positions in the right- and left-hand vicinities of the interface, respectively.

(3) The interface between the non-infected zone and the right infected zone $\left(x=\delta_{2}\right)$. At this interface, again, the continuities of both water flux and water concentration are satisfied. They are

$$
\begin{aligned}
- & D_{\mathrm{HM}}^{(0)} \frac{\partial c\left(\delta_{2-}, t\right)}{\partial x}+\frac{j}{F}\left[w_{\mathrm{HM}}^{(0)}+w_{\mathrm{HM}}^{(1)} c\left(\delta_{2-}, t\right)\right] \\
=-D_{q_{\mathrm{c}}}^{(0)} & \frac{\partial c_{\mathrm{H}_{2} \mathrm{O}}\left(\delta_{2+}, t\right)}{\partial x}+\frac{j}{F}\left[w_{q_{\mathrm{c}}}^{(0)}+w_{q_{\mathrm{c}}}^{(1)} c_{\mathrm{H}_{2} \mathrm{O}}\left(\delta_{2+}, t\right)\right],
\end{aligned}
$$

$c\left(\delta_{2-}, t\right)=c\left(\delta_{2+}, t\right)$,

where $\delta_{2+}$ and $\delta_{2-}$ account for the positions in the right- and left-hand vicinities of the interface, respectively.

(4) The interface between the right infected zone and the cathode $(x=d)$. At this interface, the water concentration is constant, namely,

$c_{\mathrm{H}_{2} \mathrm{O}}(d, t)=c_{0}$,

where $c_{0}$ is the initial water concentration of the membrane at the interface $x=d$, which is in equilibrium with the saturated vapor. This condition is justified because during the operation of a fuel cell the water is constantly generated from the reaction at the cathode so that the water concentration at the cathode can be maintained constant.

\section{The parameters used in the present analyses}

We consider the membrane made of Nafion ${ }^{\mathscr{R}} 117$ and assume the contaminant ion to be $\mathrm{Na}^{+}[26,27]$. The water diffusion coefficients of the H-type membrane are $D_{\mathrm{HM}}=D_{\mathrm{HM}}^{(0)}=1.25 \times 10^{-5} \mathrm{~cm}^{2} / \mathrm{s}\left(\right.$ at $\left.\lambda=14, T=80^{\circ} \mathrm{C}\right)$ and those of the Na-type membrane are $D_{\mathrm{NaM}}=$ $D_{\mathrm{NaM}}^{(0)}=5.0 \times 10^{-6} \mathrm{~cm}^{2} / \mathrm{s}\left(\right.$ at $\left.\lambda=14, T=60{ }^{\circ} \mathrm{C}\right)[25,36$ 39]. The water transference coefficient of the H-type membrane is $w_{\mathrm{HM}}=3.2$ (at $\lambda=22, T=80^{\circ} \mathrm{C}$ ), of the Na-type is $w_{\mathrm{NaM}}=10.2$ (at $\lambda=17, T=50{ }^{\circ} \mathrm{C}$ ) [25,29]. Since $w_{\mathrm{H}_{2} \mathrm{O}}=w^{(0)}+w^{(1)} c$, and $w^{(0)}=0$ and $w^{(1)}=$ $M V_{\mathrm{ex}} w_{\mathrm{M}} / 22 d_{\mathrm{dry}}$, so that for a H-type membrane one has $w_{\mathrm{M}}=w_{\mathrm{HM}}$ and for a Na-type membrane one has $w_{\mathrm{M}}=w_{\mathrm{NaM}}$, where $M$ is the molar mass of the membrane taken as $1100 \mathrm{~g} / \mathrm{mol}, V_{\mathrm{ex}}$ is the volume expansion 
rate of the membrane from dry to wet and is taken as 1.62 , and $d_{\mathrm{dry}}$ is the density of the dry membrane and is taken to be $2.02 \mathrm{~g} / \mathrm{cm}^{3}$ [24,30,36-39]. The water concentration in the membrane can be expressed by $c=$ $\lambda d_{\text {dry }} / M V_{\text {ex }}$. By assuming that the initial water concentration $c_{0}$ is in equilibrium with the water vapor in the membrane, or the vapor entering into the cathode is saturated, one has $\lambda=14[24,25]$. The mobility of $\mathrm{H}^{+}$in the H-type membrane is $u_{\mathrm{H}^{+}}^{0}=1.49 \times 10^{-7} \mathrm{~m}^{2} / \mathrm{V} \mathrm{s}$ and that of $\mathrm{Na}^{+}$in the Na-type membrane is $u_{\mathrm{Na}^{+}}^{0}=2.7 \times$ $10^{-8} \mathrm{~m}^{2} / \mathrm{V} \mathrm{s}$. The presence of other ions will reduce the mobility of the ion itself, so that the above two mobilities can be modified into $u_{\mathrm{H}^{+}}=u_{\mathrm{H}^{+}}^{0}\left(1-0.2 X_{\mathrm{Na}^{+}}\right)$and $u_{\mathrm{Na}^{+}}=u_{\mathrm{Na}^{+}}^{0}\left(1-0.2 X_{\mathrm{H}^{+}}\right)[34]$.

Accordingly, the ratio between the mobilities of $\mathrm{Na}^{+}$ and $\mathrm{H}^{+}$can be expressed by

$$
\begin{aligned}
p_{\mathrm{a}} & =\frac{u_{\mathrm{Na}^{+}}}{u_{\mathrm{H}^{+}}}=\frac{u_{\mathrm{Na}^{+}}^{0}\left(1-0.2 X_{\mathrm{H}^{+}}\right)}{u_{\mathrm{H}^{+}}^{0}\left(1-0.2 X_{\mathrm{Na}^{+}}\right)} \\
& =\frac{u_{\mathrm{Na}^{+}}^{0}\left[1-0.2\left(1-q_{\mathrm{a}}\right)\right]}{u_{\mathrm{H}^{+}}^{0}\left(1-0.2 q_{\mathrm{a}}\right)} \quad\left(0<x<\delta_{1}\right), \\
p_{\mathrm{c}} & =\frac{u_{\mathrm{Na}^{+}}}{u_{\mathrm{H}^{+}}}=\frac{u_{\mathrm{Na}^{+}}^{0}\left(1-0.2 X_{\mathrm{H}^{+}}\right)}{u_{\mathrm{H}^{+}}^{0}\left(1-0.2 X_{\mathrm{Na}^{+}}\right)} \\
& =\frac{u_{\mathrm{Na}^{+}}^{0}\left[1-0.2\left(1-q_{\mathrm{c}}\right)\right]}{u_{\mathrm{H}^{+}}^{0}\left(1-0.2 q_{\mathrm{c}}\right)} \quad\left(\delta_{2}<x<d\right),
\end{aligned}
$$

in which $X_{\mathrm{Na}^{+}}$is the ionic fraction of $\mathrm{Na}^{+}$in the membrane [34]. There are some other routine parameters used in the present analyses but these are not shown here. For the details of their values the reader is referred to Table 1 of Ref. [26].

\section{Results and discussion}

Based on the theoretical model and boundary and initial conditions shown in Section 2, we implement a systematic parametric study to examine the transient behavior of the water content across the membrane under the influence of various physical parameters. Special attention will be paid to the time needed for the water distribution across the membrane to reach the steady state, accounted for by $t_{\mathrm{ss}}$, and the influence on $t_{\mathrm{ss}}$ of different physical parameters is discussed. In the present paper $t_{\mathrm{ss}}$ is defined as the time at which the following relation is satisfied

$$
\operatorname{abs}\left\{\operatorname{Max}\left[y_{n+1}(i, j)-y_{n}(i, j)\right] / y_{n+1}(i, j)\right\}<10^{-4} \text {, }
$$

in which $y_{n}(i, j)$ is any physical variable at the $n$th time step on node $(i, j)$. These control parameters, include the current density $j$, the initial current density $j_{0}$, the ionic fraction of the contaminant at cathode $\left(q_{\mathrm{c}}\right)$ and anode $\left(q_{\mathrm{a}}\right)$, and the humidification parameter $k$. The values of these parameters employed in the theoretical model corresponding to the base case are $T=80{ }^{\circ} \mathrm{C}, p=1$ atm, $d=100 \mu \mathrm{m}$, and $\delta_{1} / d=\left(d-\delta_{2}\right) / d=0.25$, which are $j_{0}=0 \quad \mathrm{~A} / \mathrm{cm}^{2} j=1 \mathrm{~A} / \mathrm{cm}^{2}, k=0.001 \mathrm{~cm} / \mathrm{s}$, and $q_{\mathrm{a}}=q_{\mathrm{c}}=0$. As the physical effect of one parameter is considered, the value of this parameter is varied while the others are fixed at those shown above.

\subsection{The effect of operational current density $j$}

Fig. 2 illustrates the change of $t_{\mathrm{ss}}$ with varying current density $(j)$ for two different initial current densities $j_{0}=0$ and $0.5 \mathrm{~A} / \mathrm{cm}^{2}$. The results of both cases show that $t_{\mathrm{ss}}$ increases exponentially with $j$ and reaches a maximum $\left(t_{\mathrm{ss}} \approx 9.8 \mathrm{~s}\right.$ for $j_{0}=0 \mathrm{~A} / \mathrm{cm}^{2}, t_{\mathrm{ss}} \approx 8.2 \mathrm{~s}$ for $j_{0}=0.5 \mathrm{~A} / \mathrm{cm}^{2}$ ) after the current density is larger than 1 $\mathrm{A} / \mathrm{cm}^{2}$. The increase of $t_{\mathrm{ss}}$ with $j$ arises because the water depletion in the membrane becomes more serious when the current density is larger, leading to the water content across the membrane changing more dramatically and thus a larger $t_{\mathrm{ss}}$ is needed. When the current density is sufficiently high, say $j>1 \mathrm{~A} / \mathrm{cm}^{2}$, the chemical reaction is so strong that the water generated from the cathode is sufficient to equilibrate the water depletion in the membrane, implying that the water content in the membrane has reached a steady state so that $t_{\mathrm{ss}}$ does not change significantly with $j$. In the same figure, one can also see that $t_{\mathrm{ss}}$ decreases as $j_{0}$ increases. This is because for a larger $j_{0}$ the initial water content of the membrane is smaller, so that the time needed to reach the steady state is shorter.

Fig. 3 shows the distribution of water concentration across the membrane under three different operational current densities, $j=0.1,0.5$ and $1.0 \mathrm{~A} / \mathrm{cm}^{2}$. It is seen that the membrane becomes much drier at the anode when the current density is larger. This is because, for a larger $j$, the electro-osmotic effect, dragging water molecules from anode to cathode is more significant than the water diffusion effect pumping water molecules from

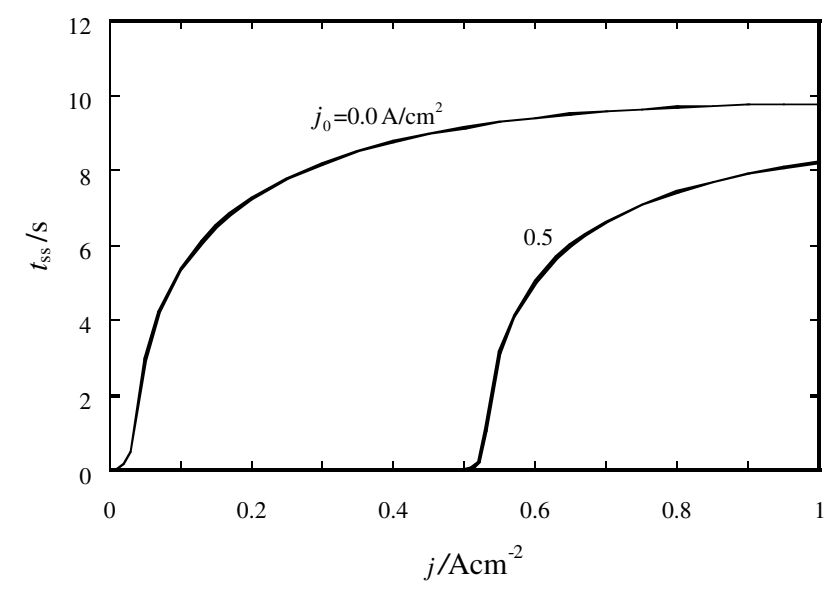

Fig. 2. The influence of operational current density $j$ on the time to reach the steady state $t_{\mathrm{ss}}$. The two curves correspond to different initial current densities. 


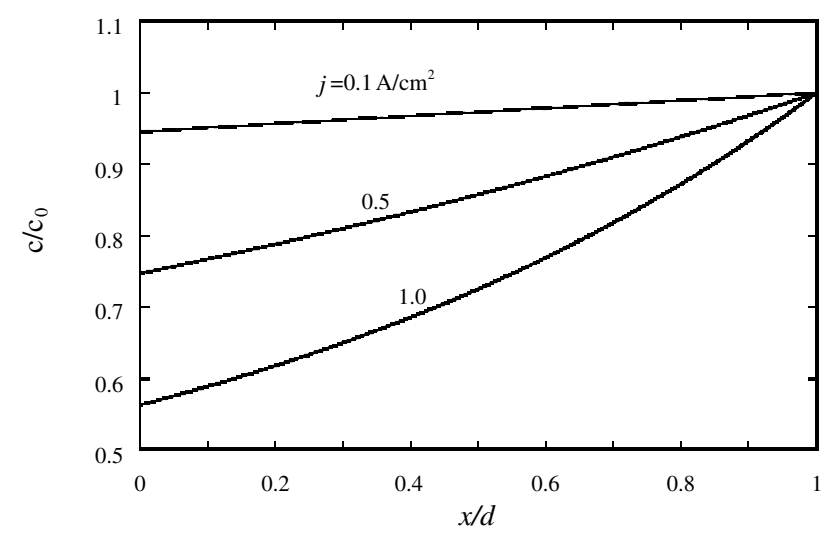

Fig. 3. Three water distributions across the membrane corresponding to three different operational current densities.

cathode to anode. This also explains why $t_{\mathrm{ss}}$ is larger for a higher $j$, as is shown in Fig. 2.

\subsection{The effect of contaminants}

Fig. 4 illustrates the variations of $t_{\mathrm{ss}}$ with $j$ for the case with contamination (curve B) and without contamination (curve A). The contaminants present at the anode and cathode are of the same concentration. It is seen that, for all the operational current densities considered, $t_{\mathrm{ss}}$ for the case with contamination, is invariably larger than that without contamination, indicating that the contaminant enhances water depletion in the membrane so that the time needed to reach the steady state is longer. That the water depletion in the membrane is enhanced by the presence of contaminant is due to the fact that the electro-osmotic coefficient of the contaminant is larger than that of $\mathrm{H}^{+}$. As a result, the amount of water dragged from anode to cathode increases, while the water diffusing from cathode to anode decreases,

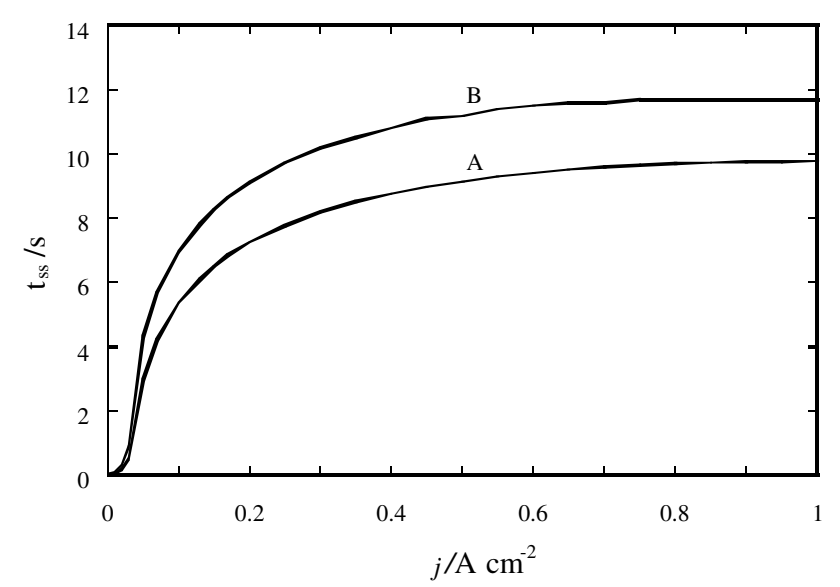

Fig. 4. The influence of the operational current density $j$ on the time to reach the steady state $t_{\mathrm{ss}}$. Curve A: without contamination $q_{\mathrm{a}}=q_{\mathrm{c}}=0$; Curve B: with contamination $q_{\mathrm{a}}=q_{\mathrm{c}}=0.5$. leading to water depletion in the membrane. Fig. 5 illustrates the distribution of the water concentration across the membrane at different times. Without contaminant, as shown in Fig. 5(a), the water concentration decreases with time in the whole membrane, the water depletion is more serious at the anode than at the cathode and the time to reach the steady state is about 9.78 s. With contaminant, as shown in Fig. 5(b), the water concentration also decreases with time in virtually the whole membrane, and the time to reach the steady state also increases to $11.7 \mathrm{~s}$. Note please that the water concentration gradient in the infected zone is greater than that in the non-infected zone, which is due to the fact that the presence of the contaminant enhances the electro-osmotic effect while it inhibits the diffusion of water. As a result, the water concentration gradient at the cathode is larger than that at the anode, implying that the impurity effect on the electro-osmosis is greater on the cathode than on the anode. We have also investigated the cases of different levels of contamination, $q_{\mathrm{a}}=q_{\mathrm{c}}=0.3$ and 0.8 , and obtained the same conclusion that a higher contaminant concentration leads to a

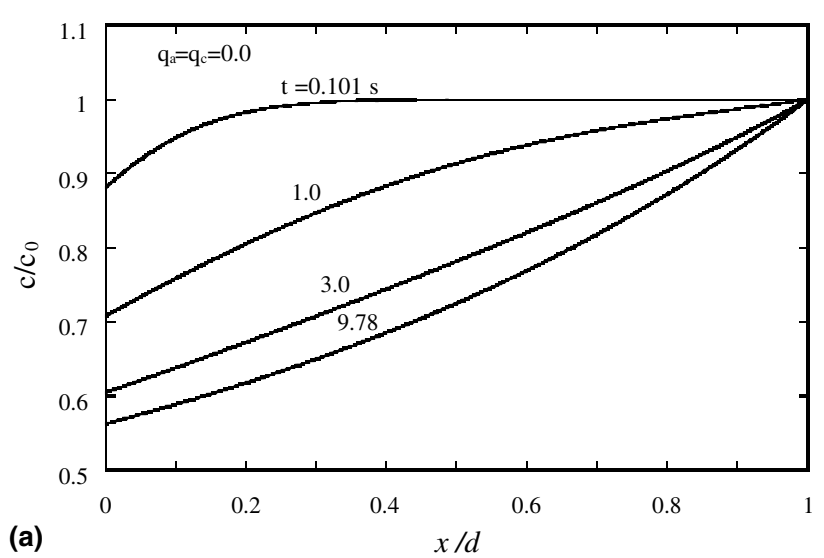

(a)

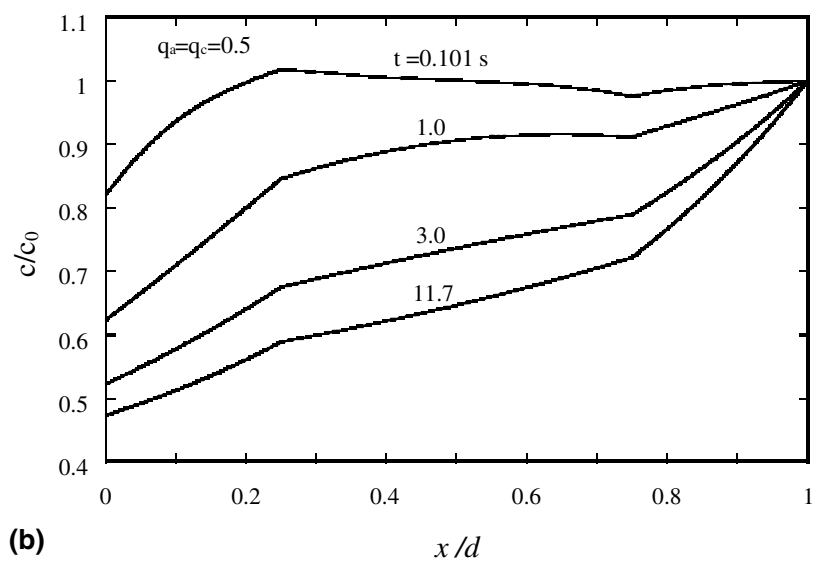

Fig. 5. The water distribution across the membrane at different times. (a) Without contamination $q_{\mathrm{a}}=q_{\mathrm{c}}=0$, corresponding to Curve A of Fig. 4. (b) With contamination $q_{\mathrm{a}}=q_{\mathrm{c}}=0.5$, corresponding to Curve B of Fig. 4. 
lower water content across the membrane, so that the time needed to reach the steady state is longer.

\subsection{The effect of initial current density $j_{0}$}

The initial current density corresponds to the water content of the membrane at the beginning of operation. As the fuel cell becomes operational, the water content in the membrane should reach the steady state in a shorter time when the initial current density is closer to the current density at the steady state. This scenario is confirmed by the results of Fig. 6, in which two curves regarding the relation between $t_{\mathrm{ss}}$ and $j_{0}$ under two different operational current densities $j=0.5$ and $1 \mathrm{~A} /$ $\mathrm{cm}^{2}$ are shown. It is seen that, as implied previously, a $j_{0}$ closer to $j$ leads to a smaller $t_{\mathrm{ss}}$. When the membrane is contaminated, as shown in Fig. 7, the presence of the

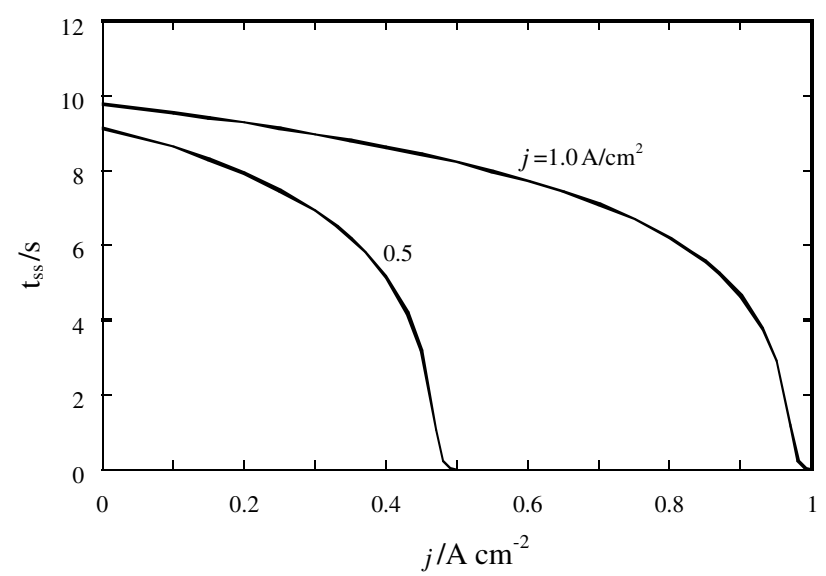

Fig. 6. The influence of initial current density $j_{0}$ on the time to reach the steady state $t_{\mathrm{ss}}$. The two curves correspond to different operational current densities.

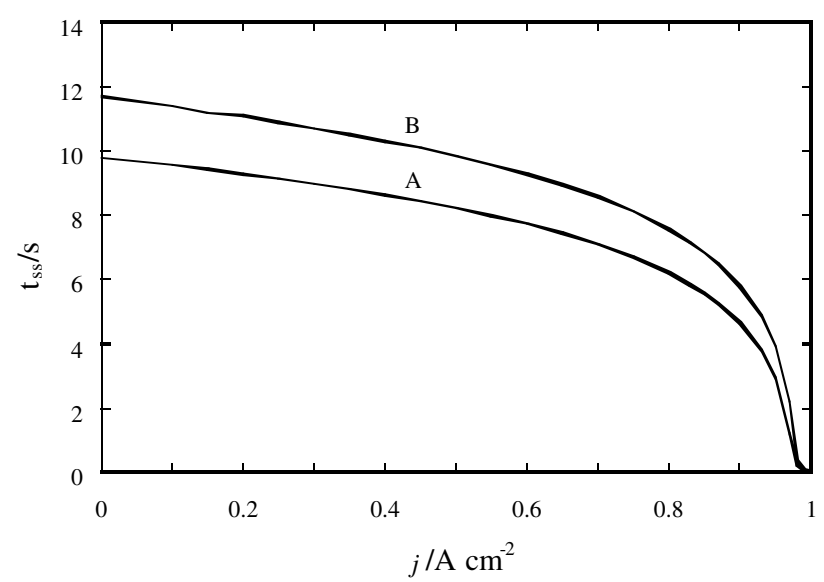

Fig. 7. The influence of the initial current density $i_{0}$ on the time to reach the steady state $t_{\mathrm{ss}}$. Curve A: without contamination $q_{\mathrm{a}}=q_{\mathrm{c}}=0$; Curve B: with contamination $q_{\mathrm{a}}=q_{\mathrm{c}}=0.5$. contaminant enhances the electro-osmotic effect, leading to a more serious water depletion at the anode. As a result, it needs a longer time for the water distribution in the membrane to reach the steady state. For both cases shown in Fig. 7, the operational current density $j$ at the steady state is approximately $1 \mathrm{~A} / \mathrm{cm}^{2}$.

\subsection{The effect of humidification parameter $k$}

The humidification parameter $k$ accounts for the amount of water entering the anode; namely, a larger $k$ implies a larger amount of water entering the anode, increases the water content of membrane, and anneals the water depletion in the membrane. This scenario is confirmed by the results of Fig. 8 that the distribution of water concentration increases for a larger $k$, so that the time $\left(t_{\mathrm{ss}}\right)$ needed to reach the steady state of the water

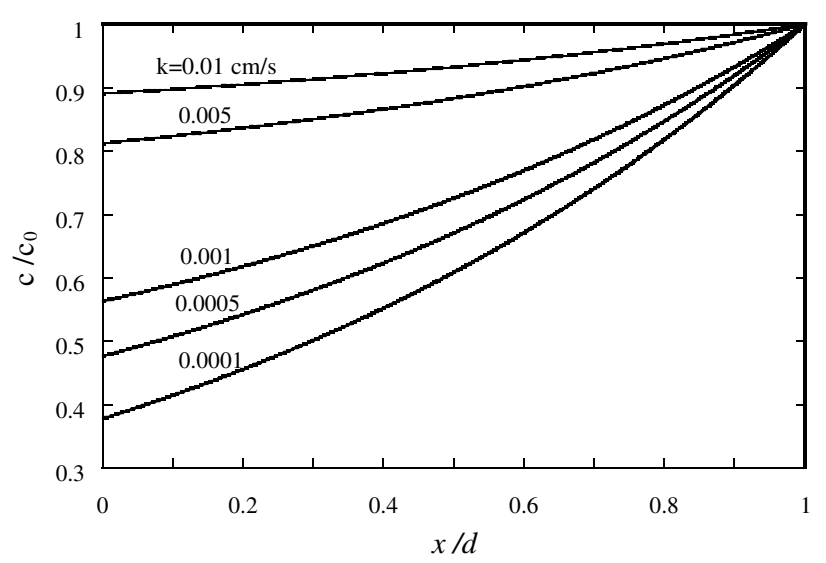

Fig. 8. The water distributions across the membrane for various humidification parameters $k$.

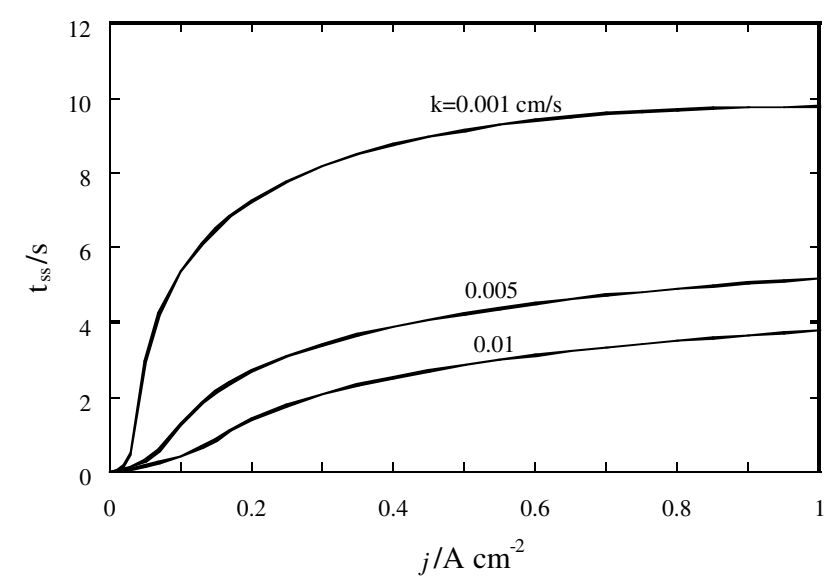

Fig. 9. The influence of the operational current density $j$ on the time to reach the steady state $t_{\mathrm{ss}}$. The three curves correspond to three different $k$. 


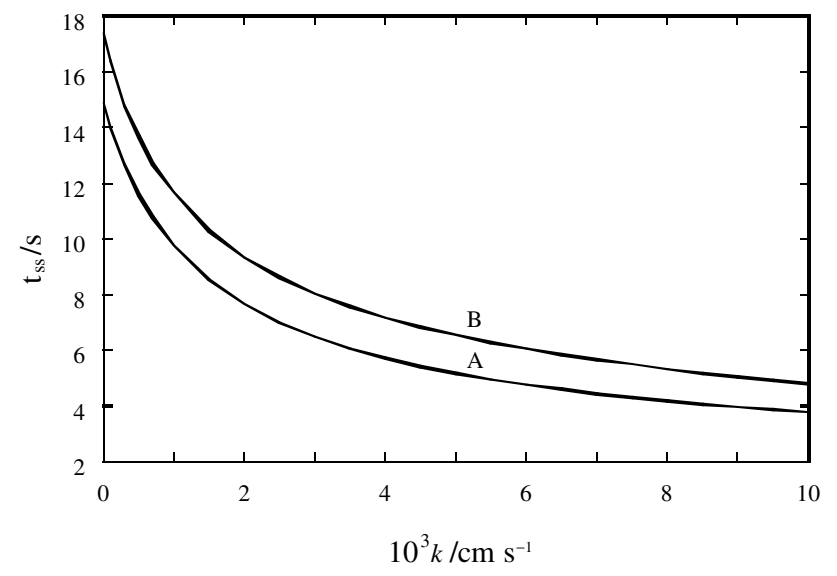

Fig. 10. The influence of humidification parameter $k$ on the time to reach the steady state $t_{\mathrm{ss}}$. Curve A: without contamination $q_{\mathrm{a}}=q_{\mathrm{c}}=0$; Curve B: with contamination $q_{\mathrm{a}}=q_{\mathrm{c}}=0.5$.

distribution in the membrane is shortened, as shown in Fig. 9. When the membrane is contaminated, as shown in Fig. 10, $t_{\mathrm{ss}}$ becomes larger because the contaminant enhances the water depletion by way of a stronger electro-osmotic effect.

\subsection{The effect of contaminants at different locations}

As we mentioned previously, the presence of contaminants will not only influence the electro-osmotic effect so that the drag of water from anode to cathode is affected, but will also inhibit the diffusion of water from cathode to anode. Since it influences the transportation of water in both directions, from anode to cathode or from cathode to anode, it is expected that the presence of contaminant in either the cathode or anode would have different levels of influence. We examine this scenario by considering four cases of different contamination situations: (a) without contamination $q_{\mathrm{a}}=q_{\mathrm{c}}=0$; (b) with contamination at the anode $q_{\mathrm{a}}=0.5, q_{\mathrm{c}}=0$; (c) with contamination at the cathode $q_{\mathrm{a}}=0, q_{\mathrm{c}}=0.5$; (d) with contamination at both the anode and cathode $q_{\mathrm{a}}=q_{\mathrm{c}}=0.5$. The results show that the presence of the contaminant at the anode (case $\mathrm{B}, t_{\mathrm{ss}}=9.86 \mathrm{~s}$ ) increases $t_{\mathrm{ss}}$ only slightly above $t_{\mathrm{ss}}$ for the case without contamination (case $\mathrm{A}, t_{\mathrm{ss}}=9.78 \mathrm{~s}$ ), implying that the influence of contaminant on the electro-osmotic effect (the mechanism driving the water from anode to cathode) is insignificant. On the other hand, the presence of contaminant at the cathode (case $\mathrm{C}, t_{\mathrm{ss}}=11.5 \mathrm{~s}$; case $\mathrm{D}$, $t_{\mathrm{ss}}=11.7 \mathrm{~s}$ ) increases $t_{\mathrm{ss}}$ to a greater extent from the case with contamination at the anode, suggesting that the contaminant reduces the diffusion of water (the mechanism driving the water from cathode to anode) more significantly. The above conclusions can be reasoned clearly from the distributions of the water concentration of these four cases as shown in Fig. 11.

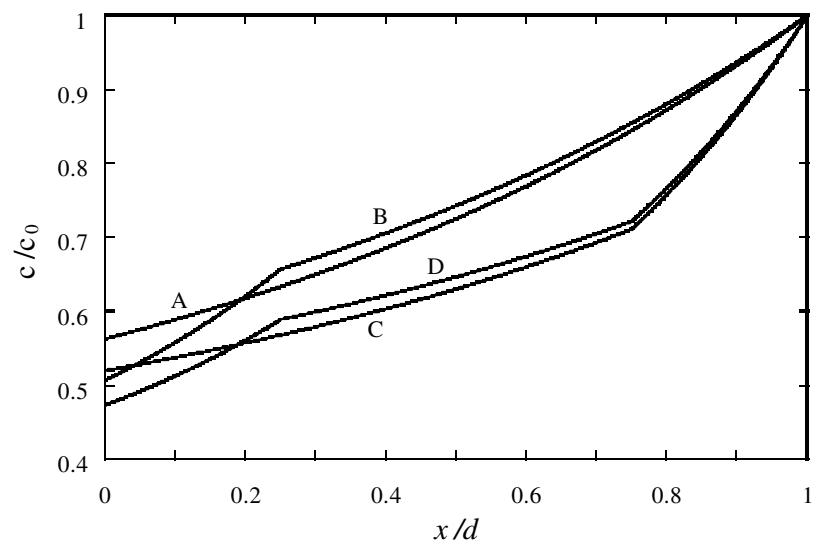

Fig. 11. The distributions of the water concentration across the membrane of the four cases (A) Without contamination $q_{\mathrm{a}}=q_{\mathrm{c}}=0$. (B) With contamination at the anode $q_{\mathrm{a}}=0.5, q_{\mathrm{c}}=0$. (C) With contamination at the cathode $q_{\mathrm{a}}=0, q_{\mathrm{c}}=0.5$. (D) With contamination at both the anode and cathode $q_{\mathrm{a}}=q_{\mathrm{c}}=0.5$.

\section{Concluding remarks}

The transient behavior of water transport across the membrane has been investigated on the basis of the theoretical model developed by Okada [26,27] and the influence of the relevant physical parameters on the time $\left(t_{\mathrm{ss}}\right)$ needed for the water distribution to reach the steady state is investigated systematically. Results from the parametric study give the following conclusions:

(1) During the fuel cell operation, water depletion at the anode is more marked than that at the cathode because the electro-osmotic effect, dragging water from anode to cathode, is usually higher than the diffusion effect pumping the water from cathode to anode. This phenomenon is more obvious when the current density is higher. As a result, $t_{\mathrm{ss}}$ increases with the current density, while the increase is annealed when the current density is sufficiently high so that the water generated from the cathode is sufficiently large that the water depletion at the anode can be balanced by the water supply from the cathode.

(2) When foreign impurity ions are present in the membrane, the contaminants enhance the electro-osmotic effect but inhibit the diffusion effect. Thus the drag of water from anode to cathode becomes larger while the diffusion of water from cathode to anode becomes smaller, both lead to a more marked depletion of water at the anode and therefore the value of $t_{\mathrm{ss}}$ increases.

(3) A higher initial current density $j_{0}$ means the water content in the membrane is smaller at the beginning of the fuel cell operation, so that the water distribution is closer to that of the steady state. Accordingly, a higher $j_{0}$ leads to a smaller $t_{\mathrm{ss}}$. 
(4) To anneal the water depletion at the anode, one can increase the humidification parameter $k$ at the anode so that the supply of water from the anode is increased. This scheme is particularly useful when the membrane is contaminated, and can be easily implemented by either increasing the humidity of the fuel at the anode side or by choosing a membrane of higher $k$.

(5) The presence of contaminant at the cathode has a greater effect on the water transport across the membrane than that at the anode, suggesting that the influence of contamination on the electro-osmotic effect is less significant than that on the diffusion effect, leading to a more marked water depletion in the membrane.

From the above concluding remarks, one can clearly see that, to have a fuel cell with an efficient dynamic response, which can be accounted for by a shorter $t_{\mathrm{ss}}$ for the water transport to reach a steady state in the membrane, one should either increase the humidity at the anode side, or choose a membrane of larger $k$ at the anode, or start the operation with a larger $j_{0}$, or prevent to a large extent the contamination in the membrane, especially at the cathode side.

\section{Acknowledgements}

The authors would like to express their grateful appreciation for financial support from the National Science Council of Taiwan through the research grants: NSC 92-2623-7-002-006-ET and NSC 91-2218-E-211-001.

\section{References}

[1] J. Larminie, A. Dicks, Fuel Cell Systems Explained, John Wiley \& Sons, Chichester, UK, 2000.

[2] P. Costamagna, S. Srinivasan, J. Power Sources 102 (2001) 253.

[3] S. Srinivasan, D.J. Manko, H. Koch, M.A. Enayetullah, A.J. Appleby, J. Power Sources 29 (1990) 367.

[4] G.A. Eisman, J. Power Sources 29 (1990) 389.

[5] H.P. Dhar, J. Electroanal. Chem. 357 (1993) 237.

[6] P. Costamagna, S. Srinivasan, J. Power Sources 102 (2001) 242.

[7] D.M. Bernardi, J. Electrochem. Soc. 137 (1990) 3344.
[8] D.M. Bernardi, M.W. Verbrugge, AIChE J. 37 (1991) 1151.

[9] D.M. Bernardi, M.W. Verbrugge, J. Electrochem. Soc. 139 (1992) 2477.

[10] T.E. Springer, T.A. Zawodzinski, S. Gottesfeld, J. Electrochem. Soc. 138 (1991) 2334.

[11] A. Rowe, X. Li, J. Power Sources 102 (2001) 82.

[12] T.V. Nguyen, R.E. White, J. Electrochem. Soc. 140 (1993) 2178.

[13] T.F. Fuller, J. Newman, J. Electrochem. Soc. 140 (1993) 1218.

[14] D. Singh, D.M. Lu, N. Djilali, Int. J. Eng. Sci. 37 (1999) 431.

[15] N. Djilali, D. Lu, Int. J. Thermal Sci. 41 (2002) 29.

[16] T. Berning, D.M. Lu, N. Djilali, J. Power Sources 106 (2002) 284.

[17] T.E. Springer, M.S. Wilson, S. Gottesfeld, J. Electrochem. Soc. 140 (1993) 3513.

[18] M. Wakizoe, O.A. Velev, S. Srinivasan, Electrochim. Acta 40 (1995) 335.

[19] J. Kim, S.M. Lee, S. Srinivasan, J. Electrochem. Soc. 142 (1995) 2670.

[20] M. Wohr, K. Bolwin, W. Schnurnberger, M. Fischer, W. Neubrand, G. Eigenberger, Int. J. Hydrogen Energy 23 (1998) 213.

[21] D.L. Wood III, J.S. Yi, T.V. Nguyen, Electrochim. Acta 43 (1998) 3795.

[22] R.F. Mann, J.C. Amphlett, Mi. A.I. Hooper, H.M. Jensen, B.A. Peppley, P.R. Roberge, J. Power Sources 86 (2000) 173.

[23] G. Maggio, V. Recupero, L. Pino, J. Power Sources 101 (2001) 275.

[24] T. Okada, G. Xie, Y. Tanabe, J. Electroanal. Chem. 413 (1996) 49.

[25] T. Okada, G. Xie, M. Meeg, Electrochim. Acta 43 (1998) 2141.

[26] T. Okada, J. Electroanal. Chem. 465 (1999) 1.

[27] T. Okada, J. Electroanal. Chem. 465 (1999) 18.

[28] G. Xie, T. Okada, Electrochim. Acta 41 (1996) 1569.

[29] G. Xie, T. Okada, J. Electrochem. Soc. 142 (1995) 3057.

[30] T. Okada, G. Xie, O. Gorseth, S. Kjelstrup, N. Nakamura, T. Arimura, Electrochim. Acta 43 (1998) 3741.

[31] T. Okada, N. Nakamura, J. Electrochem. Soc. 144 (1997) 2744.

[32] T. Okada, S. Moller-Holst, O. Gorseth, S. Kjelstrup, J. Electroanal. Chem. 442 (1998) 137.

[33] T. Okada, Y. Ayato, M. Yuasa, I. Sekine, J. Phys. Chem. B 103 (1999) 3315.

[34] T. Okada, S. Kjelstrup-Ratkje, H. Hanche-Olsen, J. Membr. Sci. 66 (1992) 179.

[35] T. Okada, S. Kjelstrup-Ratkje, S. Moller-Holst, L.O. Jerdal, K. Friestad, G. Xie, R. Holmen, J. Membr. Sci. 111 (1996) 159.

[36] T.A. Zawodzinski, J. Davey, J. Valerio, S. Gottesfeld, Electrochim. Acta 40 (1995) 297.

[37] T.A. Zawodzinski Jr., C. Derouin, S. Radzinski, R.J. Sherman, V.T. Smith, T.E. Springer, S. Gottesfeld, J. Electrochem. Soc. 140 (1993) 1041

[38] T.A. Zawodzinski Jr., T.E. Springer, J. Davey, R. Jestel, C. Lopez, J. Valerio, S. Gottesfeld, J. Electrochem. Soc. 140 (1993) 1981.

[39] T.F. Fuller, J. Newman, J. Electrochem. Soc. 139 (1992) 1332. 